\section{JTI}

JOURNAL OF

TRAUMA AND INJURY

Received: January 13, 2020

Revised: March 2, 2020

Accepted: March 16, 2020

\section{Correspondence to}

Pil Young Jung, M.D., Ph.D.

Department of Surgery, Wonju Severance Christian Hospital, Yonsei University

Wonju College of Medicine, 20 Ilsan-ro,

Wonju 26426, Korea

Tel: $+82-33-741-0882$

Fax: +82-33-741-0574

E-mail:surgery4trauma@yonsei.ac.kr

\title{
Resuscitative Endovascular Balloon Occlusion of the Aorta in Impending Traumatic Arrest: Is It Effective?
}

\author{
Jae Sik Chung, M.D. ${ }^{1}$, Oh Hyun Kim, M.D., Ph.D. ${ }^{2}$, Seongyup Kim, M.D. ${ }^{1}$, \\ Ji Young Jang, M.D. ${ }^{3}$, Gyo Jin An, M.D. ${ }^{2}$, Pil Young Jung, M.D., Ph.D. ${ }^{1}$ \\ ${ }^{1}$ Department of Surgery, Wonju Severance Christian Hospital, Yonsei University Wonju \\ College of Medicine, Wonju, Korea \\ ${ }^{2}$ Department of Emergency Medicine, Wonju Severance Christian Hospital, Yonsei \\ University Wonju College of Medicine, Wonju, Korea \\ ${ }^{3}$ Department of Surgery, National Health Insurance Service Ilsan Hospital, Goyang, Korea
}

Purpose: Hemorrhagic shock is the leading cause of death in trauma patients worldwide. Resuscitative endovascular balloon occlusion of the aorta (REBOA) is a technique used to improve the hemodynamic stability of patients with traumatic shock and to temporarily control arterial hemorrhage. However, further research is required to determine whether REBOA with cardiopulmonary resuscitation (CPR) in near-arrest or arrest trauma patients can help resuscitation. We analyzed trauma patients who underwent REBOA according to their CPR status and evaluated the effects of REBOA in arrest situations.

Methods: This study was a retrospective single-regional trauma center study conducted at a tertiary medical institution from February 2017 to November 2019. We evaluated the mortality of severely injured patients who underwent REBOA and analyzed the factors that influenced the outcome. Patients were divided into CPR and non-CPR groups. Results: We reviewed 1,596 trauma patients with shock, of whom 23 patients underwent REBOA (1.4\%). Two patients were excluded due to failure and a repeated attempt of REBOA. The Glasgow Coma Scale score was lower in the CPR group than in the nonCPR group $(p=0.009)$. Blood pressure readings at the emergency room were lower in the CPR group than in the non-CPR group, including systolic blood pressure $(p=0.012)$, diastolic blood pressure $(p=0.002)$, and mean arterial pressure $(p=0.008)$. In addition, the mortality rate was higher in the CPR group (100\%) than in the non-CPR group (50\%) $(p=0.012)$. The overall mortality rate was $76.2 \%$.

Conclusions: Our study suggests that if REBOA is deemed necessary in a timely manner, it is better to perform REBOA before an arrest occurs. Therefore, appropriate protocols, including pre-hospital REBOA, should be constructed to demonstrate the effectiveness of REBOA in reducing mortality in arrest or impending arrest patients.

Keywords: Hemorrhagic shock; Endovascular procedures; Trauma 


\section{INTRODUCTION}

Hemorrhagic shock is the leading cause of potentially preventable death in trauma patients worldwide $[1,2]$. Resuscitative endovascular balloon occlusion of the aorta (REBOA) is a technique for improving the hemodynamic stability of patients with traumatic shock, maintaining brain and coronary artery perfusion, and temporarily controlling arterial hemorrhage $[3,4]$, This procedure can also be used as an alternative to resuscitative thoracotomy [5]. A recent report discussed the use of REBOA in trauma patients and concluded that REBOA is a technically feasible method of rapid aortic occlusion for the resuscitation of patients experiencing hemorrhagic shock due to abdominal or pelvic bleeding [6,7].

REBOA is extremely time-dependent and has limited indications due to complications such as distal ischemia and mortality. Nonetheless, in the event of traumatic hemorrhagic shock, the appropriate application of REBOA can play an important role in proceeding to the next stage of treatment [8].

However, REBOA is not yet included in the standard care of most hospitals, as no clear mortality benefits have yet been shown. Further research is required to determine whether REBOA with cardiopulmonary resuscitation (CPR) in a near-arrest or arrest situation can help with resuscitation in trauma patients $[9,10]$.

Therefore, in this study, we analyzed trauma patients who underwent REBOA according to their CPR status and analyzed the effects of REBOA in arrest situations. We hypothesized that there would be a difference in the need for REBOA depending on the urgency of trauma patients.

\section{METHODS}

This retrospective study was conducted from February 2017 to November 2019 at a single regional trauma center belonging to a tertiary medical institution in the Republic of Korea. Our study was approved by the Institutional Review Board (CR319152).

\section{Population}

We used the registry of Yonsei University Wonju Severance Christian Hospital to examine data on trauma patients during the study period with Abbreviated Injury Scale (AIS) codes 4 and 5 who underwent REBOA. The patients were divided into two groups (CPR and non-CPR) for a comparative analysis. The CPR group comprised patients with arrest related to trauma who received $\mathrm{CPR}$, while the non-CPR group included their counterparts who did not receive CPR. Demographic data, Glasgow Coma Scale (GCS), the Injury Severity Score, systolic blood pressure (SBP), diastolic blood pressure (DBP), mean arterial pressure (MAP), mechanism of injury, time to REBOA from injury and emergency room (ER) arrival, duration of REBOA, type of occlusion, diameter (Fr) of the catheter used, zone of REBOA, subsequent procedures, time to the subsequent procedure from REBOA ballooning, complications, and mortality were obtained from the electronic medical records of the study patients. The GCS scores at the time of the ER visit were measured, and blood pressure (BP) was recorded at the point when the trauma surgeon decided to perform REBOA. The mechanisms of injury were classified as car traffic accidents (TA), motorcycle TA, pedestrian TA, falls, and crushing injuries. The subsequent procedures that patients underwent were classified as CPR, surgery, and angioembolization. In all cases in the CPR group, CPR was performed pre-hospital or within 10 minutes of arrival at the hospital.

\section{REBOA}

All cases collected were in-hospital cases. REBOA was performed based on the judgment of the trauma surgeon on duty when brain injury and chest injury were unclear upon the primary assessment and the patient's BP was low (SBP $\leq 80 \mathrm{mmHg}, \mathrm{MAP} \leq 60 \mathrm{mmHg}$ ). We used Reliant 12-Fr and Rescue 7-Fr catheters, in all cases, the access site was the femoral artery. The REBOA zone was confirmed via $\mathrm{X}$-ray and total or partial occlusion was performed depending on the patient's condition. The duration of REBOA was measured from the first balloon inflation to the final deflation. 


\section{Statistical analysis}

For the comparison of trauma patients who underwent REBOA with or without CPR, the Student $t$-test was used for continuous variables, based on normal distribution, which was confirmed using the Shapiro-Wilk test, and the Fisher exact test was used to compare categorical variables. Analyses were performed using SPSS version 25 (IBM Corp., Armonk, NY, USA).

\section{RESULTS}

\section{Patient selection and characteristics}

In total, 1,596 trauma patients with AIS 4 and 5 coding with shock were assessed, of whom 23 underwent REBOA (1.4\%). Two patients were excluded due to failure and a repeated attempt of REBOA. The patient flowchart is summarized in Fig. 1.

Twenty-one patients were eligible for this study. In the $\mathrm{CPR}$ group, eight patients were already receiving CPR at the time of ER arrival; six of them had experienced return of spontaneous circulation (ROSC) and immediately underwent REBOA. Three patients were in-hospital CPR cases after ER arrival. The reason for CPR was hemorrhagic shock in all cases. The GCS scores were significantly lower in the CPR group than in the non-CPR group $(p=0.009)$. The BP readings at ER arrival were lower in the CPR group, including SBP ( $p=0.012)$, DBP $(p=0.002)$, and MAP ( $p=0.008$ ). The rate of subsequent CPR was higher in the CPR group (45.5\%) and the rate of surgery was significantly higher in the non-CPR group (90\%) $(p=0.035)$. In addition, the mortality rate was $100 \%$ in the CPR group, but was lower in the non-CPR group $(50 \%)(p=0.012)$. The overall patient mortality rate was $76.2 \%$. All participants were blunt trauma patients, and those in the CPR group tended to have a shorter time to REBOA from injury and from ER arrival, but not to a statistically significant extent. The time from REBOA to the subsequent procedure for hemostasis was from 0 to 40 minutes. In the case where the delay was 0 minute, the patient received REBOA during $\mathrm{CPR}$, but $\mathrm{CPR}$ continued without ROSC. The other variables analyzed did not show significant between-group differences (Table 1).

\section{The reasons for REBOA and complications}

The most common reason for REBOA was intra-abdominal organ injury $(n=10,48 \%)$, followed by pelvic bone fracture $(n=7,33 \%)$, combined injury $(n=3,14 \%)$, and lower extremity injury $(n=1,5 \%)$ (Fig. 2). In the patients with intra-abdominal organ injury, the most commonly injured organ was the liver, followed by the inferior vena cava, mesentery, kidney, and unknown organs. All cases of combined injuries involved a pelvic bone fracture with intra-abdominal organ injury (Table 2).

Ischemic complications occurred in two patients, one with lower limb ischemia and one with bowel ischemia,

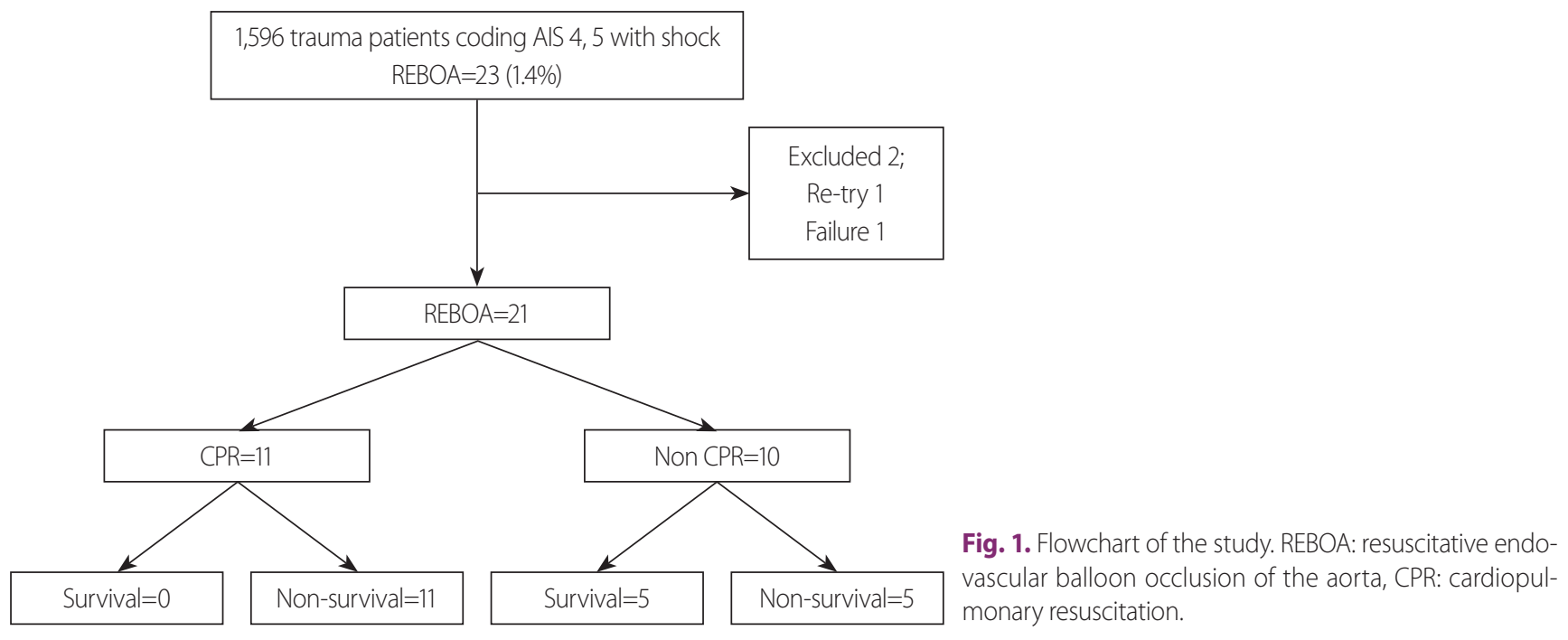


Table 1. Characteristics of trauma patients according to CPR status

\begin{tabular}{|c|c|c|c|c|}
\hline & Total $(n=21)$ & CPR (n=11) & Non-CPR $(n=10)$ & $p$-value \\
\hline Gender & & & & 0.670 \\
\hline Male & $10(47.6)$ & $6(54.5)$ & $4(40.0)$ & \\
\hline Female & $11(52.4)$ & $5(45.5)$ & $6(60.0)$ & \\
\hline Age & $51.65 \pm 18.67$ & $55.09 \pm 17.64$ & $48.20 \pm 19.73$ & 0.409 \\
\hline GCS & $6.38 \pm 3.74$ & $3.55 \pm 1.80$ & $9.20 \pm 5.37$ & 0.009 \\
\hline ISS & $29.73 \pm 13.44$ & $25.45 \pm 9.74$ & $34.00 \pm 20.52$ & 0.251 \\
\hline $\mathrm{SBP}(\mathrm{mmHg})$ & $58.92 \pm 12.36$ & $39.73 \pm 28.36$ & $66.10 \pm 8.63$ & 0.012 \\
\hline $\mathrm{DBP}(\mathrm{mmHg})$ & $23.42 \pm 13.82$ & $9.64 \pm 6.84$ & $37.20 \pm 19.05$ & 0.002 \\
\hline MAP $(\mathrm{mmHg})$ & $35.09 \pm 14.39$ & $25.27 \pm 18.21$ & $44.90 \pm 10.56$ & 0.008 \\
\hline Mechanism of injury & & & & 1.000 \\
\hline In-car TA & $6(28.6)$ & $3(27.3)$ & $3(30.0)$ & \\
\hline Fall & $5(23.8)$ & $2(18.2)$ & $3(30.0)$ & \\
\hline Motorcycle TA & $3(14.3)$ & $2(18.2)$ & $1(10.0)$ & \\
\hline Pedestrian TA & $6(28.6)$ & $3(27.3)$ & $3(30.0)$ & \\
\hline Crushing injury & $1(4.8)$ & $1(9.1)$ & $0(0.0)$ & \\
\hline Injury to REBOA (minutes) & $188.50 \pm 85.26$ & $145.00 \pm 70.85$ & $232.00 \pm 139.66$ & 0.083 \\
\hline ER to REBOA (minutes) & $48.54 \pm 37.88$ & $19.27 \pm 10.25$ & $77.80 \pm 45.50$ & 0.059 \\
\hline REBOA to subsequent procedure (minutes) & $16.66 \pm 12.15$ & $15.82 \pm 13.14$ & $17.50 \pm 11.15$ & 0.757 \\
\hline Duration (minutes) & $177.40 \pm 63.02$ & $245.91 \pm 57.38$ & $108.90 \pm 68.65$ & 0.384 \\
\hline Caliber of catheter & & & & 0.837 \\
\hline Reliant $12 \mathrm{Fr}$ & $9(42.86)$ & $6(54.5)$ & $3(30.0)$ & \\
\hline Rescue $7 \mathrm{Fr}$ & $12(57.14)$ & $5(45.5)$ & $7(70.0)$ & \\
\hline Type of occlusion & & & & 0.387 \\
\hline Total & $13(61.9)$ & $8(72.7)$ & $5(50.0)$ & \\
\hline Partial & $8(38.1)$ & $3(27.3)$ & $5(50.0)$ & \\
\hline Zone of REBOA & & & & 1.000 \\
\hline । & $10(47.6)$ & $5(45.5)$ & $5(50.0)$ & \\
\hline$\|$ & $2(9.5)$ & $1(9.1)$ & $1(10.0)$ & \\
\hline III & $9(42.9)$ & $5(45.5)$ & $4(40.0)$ & \\
\hline Following procedure & & & & 0.035 \\
\hline Ongoing CPR & $5(23.8)$ & $5(45.5)$ & $0(0.0)$ & \\
\hline Operation & $14(66.7)$ & $5(45.5)$ & $9(90.0)$ & \\
\hline Angioembolization & $2(9.5)$ & $1(9.1)$ & $1(10.0)$ & \\
\hline Complication & & & & 0.214 \\
\hline Yes & $2(9.52)$ & $2(18.2)$ & $0(0.0)$ & \\
\hline No & $19(90.48)$ & $9(81.8)$ & $10(100.0)$ & \\
\hline In-hospital mortality & & & & 0.012 \\
\hline Yes & $16(76.2)$ & $11(100.0)$ & $5(50.0)$ & \\
\hline No & $5(23.8)$ & $0(0.0)$ & $5(50.0)$ & \\
\hline
\end{tabular}

Values are presented as mean \pm standard deviation or number (\%).

GCS: Glasgow Coma Scale, ISS: Injury Severity Score, SBP: systolic blood pressure, DBP: diastolic blood pressure, MAP: mean arterial pressure, TA: traffic accident, REBOA: resuscitative endovascular balloon occlusion of the aorta, ER: emergency room, CPR: cardiopulmonary resuscitation. 
both of whom died. In the patient with lower limb ischemia, the REBOA zone was III, and partial occlusion was performed with a Reliant 12-Fr catheter. In the bowel ischemia patient, the REBOA zone was I, partial occlusion was performed, and a Rescue 7-Fr catheter was used. The

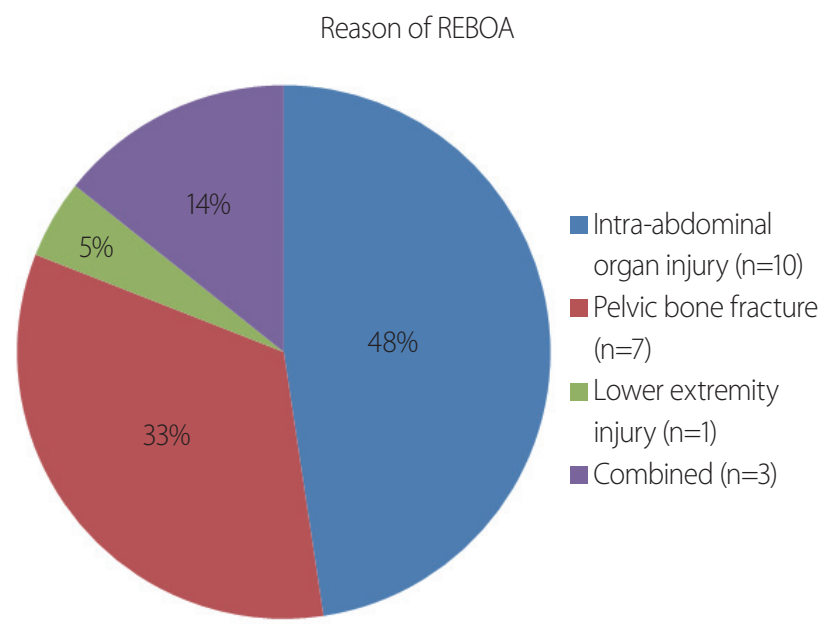

Fig. 2. Reasons for REBOA. REBOA: resuscitative endovascular balloon occlusion of the aorta.

Table 2. Reasons for REBOA

\begin{tabular}{|lc|}
\hline Reasons for REBOA & Number \\
\hline Intra-abdominal injury & 4 \\
Liver & 2 \\
IVC & 2 \\
Mesentery & 1 \\
Kidney & 1 \\
Unknown & 7 \\
Pelvic bone fracture & 1 \\
Lower extremity injury & 3 \\
Combined injury & 21 \\
Total
\end{tabular}

REBOA: resuscitative endovascular balloon occlusion of the aorta, IVC: inferior vena cava. duration of REBOA was 350 and 320 minutes for the limb ischemia and bowel ischemia patients, respectively (Table 3 ).

\section{DISCUSSION}

In our study, the variables that showed significant differences between the CPR group and non-CPR group in trauma patients who underwent REBOA were GCS score, initial $\mathrm{BP}$, the subsequent procedure, and mortality. The most common reason for REBOA was intra-abdominal organ injury.

In this study, two cases of REBOA were excluded, one because of failure and the other because REBOA was re-attempted. In the case of failure, hemorrhagic shock due to traumatic aortic rupture was suspected and emergency surgery was performed immediately after arrival at the hospital. At the same time as the operation, intraoperative REBOA was performed for bleeding control, but the catheter was not maintained because continuous $\mathrm{CPR}$ was performed for resuscitation, and the occlusion failed. The case of re-attempted REBOA was a patient with hemorrhagic shock due to a pelvic bone fracture. Pre-peritoneal pelvic packing (PPP) was performed immediately after REBOA. The patient had stable BP, REBOA was removed, the patient was transferred to the intensive care unit (ICU), and a secondary elective operation was planned. However, on the first day in the ICU, the patient showed signs of shock again. The patient underwent reinsertion of RE$\mathrm{BOA}$, and a secondary emergency operation for bleeding control was performed; nonetheless, resuscitation failed, and the patient died.

The GCS score has a clear association with prognosis in trauma patients, and can clearly document and provide characteristics of the patient's clinical status [11]. In fact, many researchers consider the GCS to be an essential tool in characterizing changes in a patient's condition and in

Table 3. Two complications after REBOA

\begin{tabular}{|lcccc|}
\hline Complication & Zone of REBOA & Catheter & Type of occlusion & Duration (minutes) \\
\hline Lower limb ischemia & II & $12 \mathrm{Fr}$ & Partial & 350 \\
Bowel ischemia & $\mathrm{I}$ & $7 \mathrm{Fr}$ & Partial & 320 \\
\hline
\end{tabular}

REBOA: resuscitative endovascular balloon occlusion of the aorta. 
deciding upon the treatment strategy, especially in patients with acute head injuries [12]. Even in the absence of head trauma, in emergency situations such as massive hemorrhage, obtaining the GCS score rapidly and accurately during the primary assessment will assist in making rapid treatment decisions, such as REBOA. In other words, the GCS provides meaningful assistance in determining the severity of trauma patients, making regular evaluation of the GCS necessary.

In this study, a significant difference was found in initial $\mathrm{BP}$ between the two groups. This was considered to be a natural result, as CPR is performed in cases of arrest or impending arrest. In the CPR group, six patients (54.5\%) underwent an additional procedure after ROSC and five patients received ongoing CPR; however, all were cases of mortality. Therefore, we report that performing REBOA in cases of arrest or impending arrest in trauma patients had no significant effect on mortality reduction. However, a point to consider is that all REBOA cases in our study were in-hospital cases. Other research has shown that successful REBOA performed pre-hospital in a traumatic arrest situation improves survival and many studies have demonstrated that pre-hospital or early REBOA is a feasible resuscitation strategy [13-15]. If early REBOA can be achieved in cases of impending arrest, this may help to improve mortality outcomes, and early REBOA should therefore be attempted on a regular basis.

According to academic societies specializing in trauma, aortic cross-clamping and PPP are the traditional approaches used to control hemorrhage during resuscitation, and are the most important methods for the temporary stabilization of severely injured trauma patients. Both methods have been widely established during the past decades. However, intra-aortic balloon occlusion, the predecessor of REBOA, was first reported by Hughes during the Korean War in 1954 and has since been widely applied in situations such as arrest, sub-diaphragmatic hemorrhage, combat casualties, and pre-hospital management [16]. In fact, according to previous studies, the mortality rate of severely injured patients who received REBOA was 47.1$90.4 \%$, which was significantly lower than those treated using other techniques, such as thoracotomy [3,4,1720]. Our study did not compare a REBOA group with a non-REBOA group, but reported a total mortality rate of
$76.2 \%$, which is not meaningfully different from the rates reported in previous studies.

In our study, there were two cases of ischemic complications, which may have been caused by catheter type, delayed occlusion time, and/or other variables. According to Teeter et al. [21] and Matsumura et al. [22], a 7-Fr introducer device for REBOA may be a safe and effective alternative to large-bore sheaths and may remain in place during the post-procedure resuscitative phase without sequelae. That is, a smaller introducer sheath for REBOA should be considered as a catheter option. With regard to the occlusion time of REBOA, although Avaro et al. [23] suggested that 40 minutes may be an optimal time, Qasim et al. [24] performed balloon occlusion for longer periods without significant procedure-related complications. Therefore, the occlusion time of REBOA does not have a clear textbook definition and should be applied appropriately depending on the situation.

There are several limitations of this study. Firstly, this study had the potential for selection bias as it was limited by several factors inherent to retrospective analyses and no randomization was performed. Therefore, a randomized prospective trial for REBOA may be more helpful to confirm the validity of the study. Secondly, the number of patients who underwent REBOA in our study was small. It is therefore difficult to generalize the results of this study. Thirdly, evaluating the GCS, or the patient's state of consciousness, is helpful in choosing whether to perform REBOA, but it would be preferable to identify injuries through appropriate studies in order to determine the causal relationships more clearly. However, most of the patients included in this study had experienced severe trauma, and therefore did not undergo a full diagnostic work-up during the initial resuscitation. Due to these limitations, additional multi-center prospective studies are required. However, despite these limitations, this study could serve as a spark for further research by Korean trauma surgeons and may help the use of REBOA to be considered in a more positive light.

\section{CONCLUSION}

REBOA was found to be associated with improved sur- 
vival in severely injured trauma patients, but it may be inadequate or have little effect on mortality in arrest situations. In other words, our study suggests that if REBOA is deemed necessary in a timely manner, it should be performed before an arrest occurs. Therefore, appropriate protocols, including pre-hospital REBOA, should be constructed to demonstrate the effectiveness of REBOA in reducing mortality in arrest or impending arrest patients.

\section{ACKNOWLEDGEMENTS}

We would like to thank Editage (www.editage.co.kr) for English language editing.

\section{REFERENCES}

1. Tien HC, Spencer F, Tremblay LN, Rizoli SB, Brenneman FD. Preventable deaths from hemorrhage at a level I Canadian trauma center. J Trauma 2007;62:142-6.

2. Sanddal TL, Esposito TJ, Whitney JR, Hartford D, Taillac PP, Mann NC, et al. Analysis of preventable trauma deaths and opportunities for trauma care improvement in utah. J Trauma 2011;70:970-7.

3. Brenner M, Inaba K, Aiolfi A, DuBose J, Fabian T, Bee T, et al. Resuscitative Endovascular balloon occlusion of the aorta and resuscitative thoracotomy in select patients with hemorrhagic shock: early results from the American Association for the Surgery of Trauma's Aortic Occlusion in resuscitation for trauma and acute care surgery registry. J Am Coll Surg 2018;226:730-40.

4. Darrabie MD, Croft CA, Brakenridge SC, Mohr AM, Rosenthal MA, Mercier NR, et al. Resuscitative endovascular balloon occlusion of the aorta: implementation and preliminary results at an academic level I trauma center. J Am Coll Surg 2018;227:12733.

5. Moore LJ, Brenner M, Kozar RA, Pasley J, Wade CE, Baraniuk MS, et al. Implementation of resuscitative endovascular balloon occlusion of the aorta as an alternative to resuscitative thoracotomy for noncompressible truncal hemorrhage. J Trauma Acute Care Surg 2015;79:523-30; discussion 530-2.

6. Brenner ML, Moore LJ, DuBose JJ, Tyson GH, McNutt MK, Albarado RP, et al. A clinical series of resuscitative endovascular balloon occlusion of the aorta for hemorrhage control and re- suscitation. J Trauma Acute Care Surg 2013;75:506-11.

7. Martinelli T, Thony F, Decléty P, Sengel C, Broux C, Tonetti J, et al. Intra-aortic balloon occlusion to salvage patients with life-threatening hemorrhagic shocks from pelvic fractures. J Trauma 2010;68:942-8.

8. Morrison JJ, Galgon RE, Jansen JO, Cannon JW, Rasmussen TE, Eliason JL. A systematic review of the use of resuscitative endovascular balloon occlusion of the aorta in the management of hemorrhagic shock. J Trauma Acute Care Surg 2016;80:324-34.

9. Tiba MH, McCracken BM, Cummings BC, Colmenero CI, Rygalski CJ, Hsu CH, et al. Use of resuscitative balloon occlusion of the aorta in a swine model of prolonged cardiac arrest. Resuscitation 2019;140:106-12.

10. Özkurtul O, Staab H, Osterhoff G, Ondruschka B, Höch A, Josten C, et al. Technical limitations of REBOA in a patient with exsanguinating pelvic crush trauma: a case report. Patient Saf Surg 2019;13:25.

11. Mattei TA, Teasdale GM. The story of the development and adoption of the Glasgow Coma Scale: part I, the early years. World Neurosurg 2019;134:311-22.

12. Teasdale G, Maas A, Lecky F, Manley G, Stocchetti N, Murray G. The Glasgow Coma Scale at 40 years: standing the test of time. Lancet Neurol 2014;13:844-54.

13. Lendrum R, Perkins Z, Chana M, Marsden M, Davenport R, Grier G, et al. Pre-hospital resuscitative endovascular balloon occlusion of the aorta (REBOA) for exsanguinating pelvic haemorrhage. Resuscitation 2019;135:6-13.

14. Matsumura Y, Matsumoto J, Kondo H, Idoguchi K, Ishida T, Okada $Y$, et al. Early arterial access for resuscitative endovascular balloon occlusion of the aorta is related to survival outcome in trauma. J Trauma Acute Care Surg 2018;85:507-11.

15. Henry R, Matsushima K, Henry RN, Wong V, Warriner Z, Strumwasser A, et al. Who would have benefited from the prehospital use of resuscitative endovascular balloon occlusion of the aorta (REBOA)? An autopsy study. J Am Coll Surg 2019;229:383-8.e1.

16. Hughes CW. Use of an intra-aortic balloon catheter tamponade for controlling intra-abdominal hemorrhage in man. Surgery 1954;36:65-8

17. Abe T, Uchida M, Nagata I, Saitoh D, Tamiya N. Resuscitative endovascular balloon occlusion of the aorta versus aortic cross clamping among patients with critical trauma: a nationwide cohort study in Japan. Crit Care 2016;20:400.

18. Brenner M, Teeter W, Hoehn M, Pasley J, Hu P, Yang S, et al. Use 
of resuscitative endovascular balloon occlusion of the aorta for proximal aortic control in patients with severe hemorrhage and arrest. JAMA Surg 2018;153:130-5.

19. Manzano-Nunez R, Naranjo MP, Foianini E, Ferrada P, Rincon E, García-Perdomo HA, et al. A meta-analysis of resuscitative endovascular balloon occlusion of the aorta (REBOA) or open aortic cross-clamping by resuscitative thoracotomy in non-compressible torso hemorrhage patients. World J Emerg Surg 2017;12:30.

20. Matsumoto S, Hayashida K, Akashi T, Jung K, Sekine K, Funabiki $\mathrm{T}$, et al. Resuscitative endovascular balloon occlusion of the aorta (REBOA) for severe torso trauma in Japan: a descriptive study. World J Surg 2019;43:1700-7.

21. Teeter WA, Matsumoto J, Idoguchi K, Kon Y, Orita T, Funabiki $\mathrm{T}$, et al. Smaller introducer sheaths for REBOA may be asso- ciated with fewer complications. J Trauma Acute Care Surg 2016;81:1039-45.

22. Matsumura Y, Matsumoto J, Kondo H, Idoguchi K, Ishida T, Kon Y, et al. Fewer REBOA complications with smaller devices and partial occlusion: evidence from a multicentre registry in Japan. Emerg Med J 2017;34:793-9.

23. Avaro JP, Mardelle V, Roch A, Gil C, de Biasi C, Oliver M, et al. Forty-minute endovascular aortic occlusion increases survival in an experimental model of uncontrolled hemorrhagic shock caused by abdominal trauma. J Trauma 2011;71:720-5; discussion 725-6.

24. Qasim Z, Brenner M, Menaker J, Scalea T. Resuscitative endovascular balloon occlusion of the aorta. Resuscitation 2015;96:275-9. 